\title{
NORMAL SELF-INTERSECTIONS OF THE CHARACTERISTIC VARIETY
}

\author{
BY RICHARD B. MELROSE ${ }^{1}$
}

Communicated by I. M. Singer, May 27, 1975

Let $P=P_{1} P_{2}+Q$ be a linear partial differential operator on $\mathbf{R}^{N}$ with $P_{1}$ and $P_{2}$, of orders $m_{1}$ and $m_{2}$, respectively, strictly hyperbolic with respect to the first variable and $Q$ of order $m_{1}+m_{2}-2$. Although the characteristic variety of $P$ may have self-intersections, the hyperbolicity of $P_{1}$ and $P_{2}$ implies local solvability for $P u=f$; indeed the Cauchy problem for $P$ is locally solvable. In this note we shall consider the propagation of singularities near the simplest type of point $z_{0} \in T^{*} \mathbf{R}^{N} \backslash 0$ where the principal symbol $p=p_{1} p_{2}$ of $P$ has a multiple zero.

We shall suppose that the characteristic varieties $A\left(P_{1}\right)$ and $A\left(P_{2}\right)$ of $P_{1}$ and $P_{2}$ intersect normally at $z_{0}$, that is, $d p_{1}\left(z_{0}\right)$ and $d p_{2}\left(z_{0}\right)$ are linear independent. In addition, it will be assumed that the Poisson bracket $\left\{p_{1}, p_{2}\right\}\left(z_{0}\right) \neq 0$. This latter assumption means that the Hamiltonian vector fields $H_{p_{1}}$ and $H_{p_{2}}$ are not tangent to $A\left(P_{1}\right) \cap A\left(P_{2}\right)$ at $z_{0}$. So, the two forward pointing bicharacteristics (of $p_{1}$ and $p_{2}$ ) through $z_{0}$ consist, near $z_{0}$, of nonsingular points of $A(P)$, except for $z_{0}$ itself. Let these curves be denoted by $c_{i}$; $\mathbf{R} \supset I \ni \rho \rightarrow c_{i}(\rho)$ where $I$ is an open interval containing $0, c_{i}(0)=z_{0}$ and $\left(c_{i}\right)_{*}(d / d \rho)=H_{p_{i}}$. It will be assumed that $I$ is chosen so small that

$$
c_{i}(I) \cap A\left(P_{j}\right)=\left\{z_{0}\right\}, \quad i \neq j .
$$

If $I^{+}\left(I^{-}\right)$is the open interval consisting of the positive (negative) points in $I$ then, by Hörmander's Theorem [4, Theorem 3.2.1], if $u \in D^{\prime}\left(\mathbf{R}^{N}\right), z_{0} \notin W F(P u)$ and $I$ is chosen so small that

$$
c_{i}(I) \cap W F(P u)=\varnothing, \quad i=1,2,
$$

then either $c_{i}\left(I^{ \pm}\right) \subset W F(u)$ or $c_{i}\left(I^{ \pm}\right) \cap W F(u)=\varnothing$ separately for the four choices of sign and bicharacteristic. Hörmander's Theorem does not, however, give any information as to whether $z_{0} \in W F(u)$ or not.

THEOREM. Suppose $A\left(P_{1}\right)$ and $A\left(P_{2}\right)$ intersect normally at $z_{0}$ and that $\left\{p_{1}, p_{2}\right\}\left(z_{0}\right) \neq 0$. If $u \in D^{\prime}\left(\mathbf{R}^{N}\right), z_{0} \notin W F(P u)$ and $I$ is chosen so small that (1) and (2) hold, then either $c_{i}\left(I^{+}\right) \cap W F(u)=\varnothing$ for $i=1,2$, or $c_{i}\left(I^{-}\right) \cap W F(u)=$ $\varnothing$ for $i=1,2$ implies $z_{0} \notin W F(u)$ and $c_{i}(I) \cap W F(u)=\varnothing$ for $i=1,2$.

AMS (MOS) subject classifications (1970). Primary 35D10, 35P20.

${ }^{1}$ This research, carried out at MIT, was supported in part by a grant from the Science Research Council. 
The main part of the proof consists in the construction of suitable microlocalizing pseudodifferential operators and this is carried out by a modification of the method used in Nirenberg's paper [5] .

Similar results hold for first order symmetric hyperbolic systems and so lead to generalized Poisson relations for the spectral measure of the associated elliptic operator (compare Chazarain [1], Duistermaat and Guillemin [2]). Let $A$ be an $m \times m$ first order system of classical elliptic pseudodifferential operators defined, for simplicity, on the $m$-fold direct sum of the half-density bundle over a compact manifold $M$ and suppose that the principal symbol $a$ of $A$ is symmetric and uniformly diagonalizable (i.e. has eigenvalues and eigenvectors smoothly defined on $\left.T^{*} M \backslash 0\right)$. Suppose further that at each point $z \in T^{*} M \backslash 0$ either $a$ has $m$ distinct eigenvalues or $m-1$ distinct eigenvalues and the coincident eigenvalues $\lambda_{i}(z)=$ $\lambda_{j}(z), i \neq j$, satisfy $d \lambda_{i}(z) \neq d \lambda_{j}(z)$ and $\left\{\lambda_{i}, \lambda_{j}\right\}(z) \neq 0$. Then the spectral density of $A$,

$$
\sigma(\mu)=\sum_{k} \delta\left(\mu-\mu_{k}\right)
$$

where $\left\{\mu_{k}\right\}$ are the eigenvalues of $A$, satisfies the following

THEOREM. The singularities of the Fourier transform $\hat{\sigma}(t)$ of $\sigma$ occur only at points $|t|=T$ such that there exists a piecewise smooth closed curve of parameter length $T$ each of whose smooth segments is an integral curve of one of the Hamiltonian fields $H_{\lambda_{j}}$.

The proofs of these and other related results will appear elsewhere. It should be noted that the proof is not constructive and does not, for example, produce a microlocal parametrix for $P$ or $\partial_{t}-A$ from which the singularities can be computed directly (see, however, Guillemin [3]).

The author wishes to thank Victor Guillemin, David Schaeffer and Gunther Uhlmann for helpful conversations.

\section{REFERENCES}

1. J. Chazarain, Formule de Poisson pour les varietes riemannienes, Invent. Math. 24 (1974), 65-82.

2. J. J. Duistermaat and V. Guillemin, The spectrum of positive elliptic operators and periodic bicharacteristics, Invent. Math. (to appear).

3. V. Guillemin, Singular symbols (manuscript) (1974).

4. L. Hörmander, On the existence and the regularity of solutions of linear pseudodifferential equations, Enseignement Math. (2) 17 (1971), 99-163. MR 48 \#9458.

5. L. Nirenberg, Lectures on linear partial differential equations, Regional Conf. Series in Math., no. 17, Amer. Math. Soc., Providence, R. I., 1973.

ST. JOHN'S COLLEGE, CAMBRIDGE, ENGLAND 